\title{
Laboratory Studies on the Removal of Radon-Born Lead from KamLAND's Organic Liquid Scintillator
}

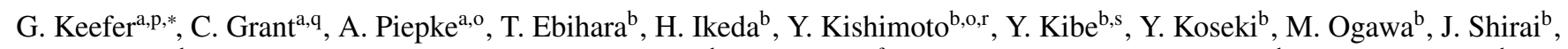

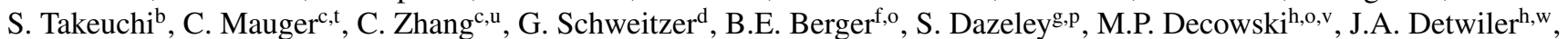
Z. Djurcic ${ }^{\mathrm{a}, \mathrm{x}}$, D.A. Dwyer ${ }^{\mathrm{c}, \mathrm{h}}$, Y. Efremenko ${ }^{\mathrm{i}}$, S. Enomoto ${ }^{\text {b,o,w }}$, S.J. Freedman ${ }^{\text {h,o, }}$, B.K. Fujikawa ${ }^{\text {h,o }}$, K. Furuno ${ }^{\mathrm{b}}$, A. Gando ${ }^{\mathrm{b}}$,

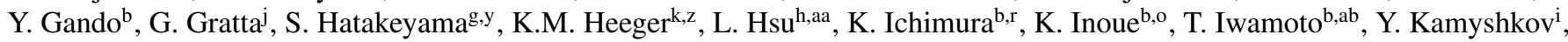
H.J. Karwowski ${ }^{\mathrm{m}}$, M. Koga ${ }^{\mathrm{b}, \mathrm{o}}$, A. Kozlov ${ }^{\text {b,o }}$, C.E. Lane ${ }^{\mathrm{n}}$, J.G. Learned ${ }^{\mathrm{e}}$, J. Maricic ${ }^{\mathrm{n}}$, D.M. Markoff ${ }^{\mathrm{m}}$, S. Matsuno ${ }^{\mathrm{e}}$, D. McKee ${ }^{1, \text { ad }}$, R.D. McKeown ${ }^{\text {c,ae }}$, T. Miletic ${ }^{\text {, }}$, T. Mitsui ${ }^{\mathrm{b}}$, M. Motoki ${ }^{\mathrm{b}}$, K. Nakajima ${ }^{\mathrm{b}, \mathrm{ai}}$, K. Nakajima ${ }^{\mathrm{b}, \mathrm{af}}$, K. Nakamura ${ }^{\mathrm{b}, \mathrm{o}}$, T. O’Donnell $^{\mathrm{h}}$,

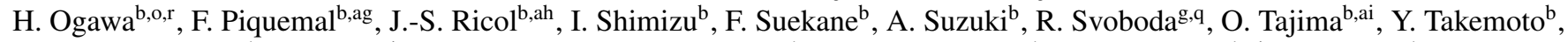
K. Tamae ${ }^{\mathrm{b}}$, K. Tolich ${ }^{\mathrm{j}}$, W. Tornow ${ }^{\mathrm{m}}$, Hideki Watanabe ${ }^{\mathrm{b}}$, Hiroko Watanabe $^{\mathrm{b}}$, L.A. Winslow ${ }^{\text {h,aj }}$, S. Yoshida $^{\text {b,ac }}$

\author{
${ }^{a}$ Department of Physics and Astronomy, University of Alabama, Tuscaloosa, Alabama 35487, USA \\ ${ }^{b}$ Research Center for Neutrino Science, Tohoku University, Sendai 980-8578, Japan \\ ${ }^{c}$ W. K. Kellogg Radiation Laboratory, California Institute of Technology, Pasadena, California 91125, USA \\ ${ }^{d}$ Department of Chemistry, University of Tennessee, Knoxville, Tennessee 37996, USA \\ ${ }^{e}$ Department of Physics and Astronomy, University of Hawaii at Manoa, Honolulu, Hawaii 96822, USA \\ ${ }^{f}$ Department of Physics, Colorado State University, Fort Collins, Colorado 80523, USA \\ ${ }^{g}$ Department of Physics and Astronomy, Louisiana State University, Baton Rouge, Louisiana 70803, USA \\ ${ }^{h}$ Physics Department, University of California, Berkeley and Lawrence Berkeley National Laboratory, Berkeley, California 94720, USA \\ ${ }^{i}$ Department of Physics and Astronomy, University of Tennessee, Knoxville, Tennessee 37996, USA \\ ${ }^{j}$ Physics Department, Stanford University, Stanford, California 94305, USA \\ ${ }^{k}$ Department of Physics, University of Wisconsin at Madison, Madison, Wisconsin 53706, USA \\ ${ }^{l}$ Department of Physics, Kansas State University, Manhattan, Kansas 66506, USA \\ ${ }^{m}$ Triangle Universities Nuclear Laboratory, Durham, North Carolina 27708, USA and Physics Departments at Duke University, North Carolina State University, \\ and the University of North Carolina at Chapel Hill, North Carolina, USA \\ ${ }^{n}$ Physics Department, Drexel University, Philadelphia, Pennsylvania 19104, USA \\ ${ }^{o}$ Kavli Institute for the Physics and Mathematics of the Universe (WPI), University of Tokyo, Kashiwa 277-8583, Japan \\ ${ }^{p}$ Present address: Lawrence Livermore National Laboratory, Livermore, California 94550, USA \\ ${ }^{q}$ Present address: Department of Physics, University of California Davis, Davis, CA 95616, USA \\ ${ }^{r}$ Present address: Kamioka Observatory, Institute for Cosmic Ray Research, University of Tokyo, Gifu 506-1205, Japan \\ ${ }^{s}$ Present address: Department of Physics, Okayama University, Okayama 700-8530, Japan \\ ${ }^{t}$ Present address: Los Alamos National Laboratory, Los Alamos, New Mexico 87545, USA \\ uresent address: Physics Department, Brookhaven National Laboratory, Upton, New York 11973, USA \\ ${ }^{v}$ Present address: Nikhef and the University of Amsterdam, Science Park, Amsterdam, Netherlands \\ ${ }^{w}$ Present address: Center for Experimental Nuclear Physics and Astrophysics, University of Washington, Seattle, Washington 98195, USA \\ ${ }^{x}$ Present address: Argonne National Laboratory, Argonne, Illinois 60439, USA \\ ${ }^{y}$ Present address: J-PARC Center, 2-4 Shirane Shirakata, Tokai-mura, Naka-gun, Ibaraki 319-1195, Japan \\ ${ }^{z}$ Present address: Department of Physics, Yale University, New Haven, Connecticut 06520, USA \\ ${ }^{a}$ Present address: Fermi National Accelerator Laboratory, Batavia, Illinois 60510, USA \\ ${ }^{a b}$ Present address: ICEPP, University of Tokyo 7-3-1 Hongo, Bunkyo-ku, Tokyo 113-0033, Japan \\ ${ }^{a c}$ Present address: Graduate School of Science, Osaka University, Toyonaka 560-0043, Japan \\ ${ }^{a d}$ Present address: Department of Chemical and Physical Sciences, Missouri Southern State University, Joplin, Missouri 64801, USA \\ ${ }^{a}$ Present address: Thomas Jefferson National Accelerator Facility, Newport News, Virginia 23606, USA \\ af Present address: Research Center for Nuclear Physics, Osaka University, Ibaraki 567-0047, Japan \\ ag Present address: CEN Bordeaux-Gradignan, CNRS/IN2P3 and Université de Bordeaux I, F-33175 Gradignan Cedex, France \\ ${ }^{a h}$ Present address: LPSC Université Joseph Fourier, CNRS/IN2P3, Institut Polytechnique de Grenoble, Grenoble, France \\ ai Present address: High Energy Accelerator Research Organization, KEK, Tsukuba 305-0801, Japan \\ ${ }^{a j}$ Present address: Department of Physics and Astronomy, University of California Los Angeles, Los Angeles, CA 90095, USA
}

\begin{abstract}
The removal of radioactivity from liquid scintillator has been studied in preparation of a low background phase of KamLAND. This paper describes the methods and techniques developed to measure and efficiently extract radon decay products from liquid scintillator. We report the radio-isotope reduction factors obtained when applying various extraction methods. During this study, distillation was identified as the most efficient method for removing radon-born lead from liquid scintillator.
\end{abstract}

Keywords: KamLAND, low background, purification, liquid scintillator, solar neutrinos 2000 MSC: 85-05 


\section{Introduction}

Sensitive detection of trace radioactivity is important for low energy solar neutrino, dark matter, and neutrinoless double beta decay experiments. Due to demanding constraints on detector backgrounds it has become essential to better understand the mechanisms that can be used to remove trace-level radioactivity from detector components, specifically from the active volume. This study was motivated by the goal of expanding the physics capability of the KamLAND experiment to include detection of low energy solar neutrinos and other events from rare physics phenomena.

The KamLAND detector is situated in the Kamioka mine, Gifu prefecture, Japan and was commissioned in 2002 to test the large mixing angle (LMA) solution of the solar neutrino problem [1, 2]. KamLAND demonstrated the validity of the LMA solution by observing the disappearance of anti-neutrinos produced in nuclear reactors in Japan [3-6]. KamLAND's active target is 1 kton of organic liquid scintillator (LS), composed of $80.2 \% n$-dodecane, $19.8 \%$ 1,2,4-trimethylbenzene (PC) and $1.36 \pm 0.03 \mathrm{~g} / \mathrm{L}$ of 2,5-diphenyloxazole (PPO).

The statistical analysis of KamLAND's low energy event distribution is performed by fitting summed beta spectra of different radionuclides to KamLAND's singles data (events not requiring delayed coincidence) and allowing their normaliza- ${ }_{53}$ tions to float freely. The result of this procedure is depicted 54 in Fig. 1 together with the data. The mass concentrations of ${ }_{55}$ the contaminants appearing in Fig. 1 are listed along with sev- ${ }_{56}$ eral others in Table 1 [7]. Analysis of the singles data yields ${ }_{57}$ an integral event rate of $3.7 \times 10^{7} \mathrm{kton}^{-1} \mathrm{day}^{-1}$ in the energy ${ }_{58}$ window of interest $([0.25,0.8] \mathrm{MeV})$. This rate is to be com- ${ }_{59}$ pared to the expected ${ }^{7} \mathrm{Be}$ solar neutrino elastic scattering sig- 60 nal of 291 events kton ${ }^{-1}$ day $^{-1}$ (calculated using BS05 solar ${ }_{61}$ rates [8], Sec. 5.5 of [7] provides detailed calculations of these ${ }_{62}$ rates). Spectral analysis allows the identification of the princi- ${ }_{63}$ pal contributors to KamLAND's background to be ${ }^{85} \mathrm{Kr},{ }^{210} \mathrm{Bi}_{64}$ and ${ }^{210} \mathrm{Po}$, the latter two supported by the decay of long-lived ${ }_{65}$ ${ }^{210} \mathrm{~Pb}$ [7]. ${ }^{85} \mathrm{Kr}$ is a fission product, found in the atmosphere mainly due to nuclear fuel re-processing [9], while ${ }^{210} \mathrm{Bi}$ and 66 ${ }^{210} \mathrm{Po}$ are ${ }^{222} \mathrm{Rn}$ decay daughters. As both ${ }^{85} \mathrm{Kr}$ and ${ }^{222} \mathrm{Rn}$ are airborne, it was assumed that these contaminants were intro- ${ }^{67}$ duced into the KamLAND LS during filling from exposure to ${ }^{68}$ air. The initial growth of ${ }^{210} \mathrm{Po}$, observed in early KamLAND data, supports this hypothesis [10]. After the initial growth the ${ }^{210}$ Po activity leveled off, indicating that it was supported by its long-lived parent ${ }^{210} \mathrm{~Pb}$. The analysis of delayed ${ }^{214} \mathrm{Bi}$ ${ }^{214} \mathrm{Po}$ beta-alpha coincidences, which occur above ${ }^{210} \mathrm{~Pb}$ in the U-decay chain, provided an effective ${ }^{238} \mathrm{U}$ decay rate of $3.7 \pm 0.5$ $\mathrm{kton}^{-1}$ day $^{-1}$. This excludes the possibility that ${ }^{210} \mathrm{Po}$, observed with an event rate of $4.6 \times 10^{6} \mathrm{kton}^{-1} \mathrm{day}^{-1}$, was supported by either ${ }^{238} \mathrm{U}$ or ${ }^{226} \mathrm{Ra}$. Although there was no direct spectroscopic evidence for the presence of ${ }^{39}$ Aractivity (an atmospheric spallation product), its existence was suspected because

${ }^{*}$ Corresponding author at: Lawrence Livermore National Laboratory, Livermore, California 94550, USA Tel.: +1 925424 5094; Fax: +1 9254245512

Email address: gregkeef er@llnl.gov (G. Keefer)

${ }^{1}$ Deceased.

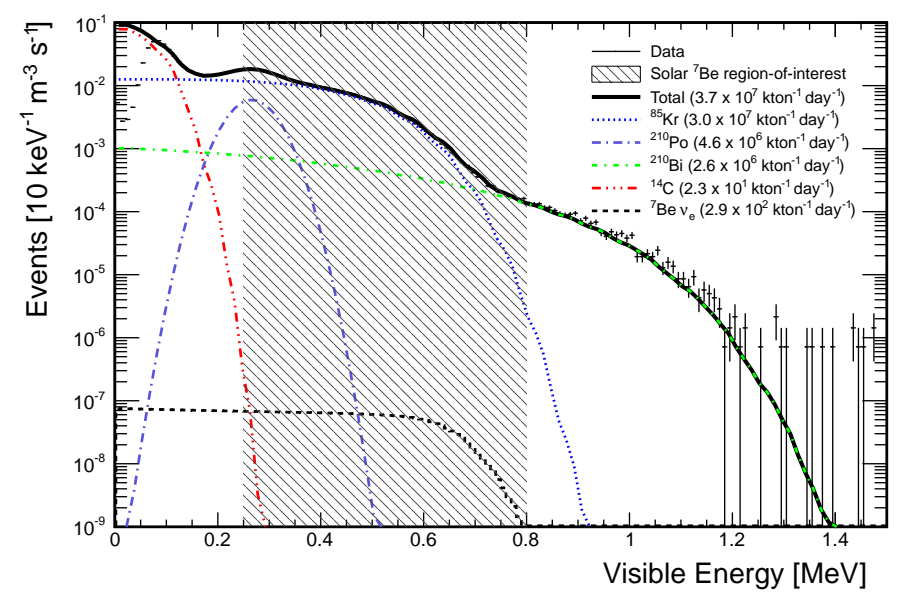

Figure 1: KamLAND low energy singles spectrum. The data is displayed together with a background model. The major background components are indicated along with their integral rates inside the energy interval $[0.25,0.8] \mathrm{MeV}$ (hatched region). For KamLAND LS $1 \mathrm{~m}^{-3} \mathrm{~s}^{-1}=1.1 \times 10^{8} \mathrm{kton}^{-1} \mathrm{day}^{-1}$. The expected ${ }^{7}$ Be solar neutrino recoil event spectrum (calculated using BS05 solar rates [8]) is overlaid to provide the appropriate scale. All spectra are converted to visible energy with KamLAND's energy response function and folded with a $\sigma=7.8 \% / \sqrt{E[\mathrm{MeV}]}$ Gaussian energy resolution [7].

of the air exposure hypothesis, and a limit on its concentration is included for completeness in Table 1.

The studies presented in this paper were undertaken to understand if and how the internal background rate, five orders of magnitude larger than the solar neutrino signal, can be sufficiently reduced by means of chemical purification. At the fundamental level, removal of impurities can be achieved by differences in chemical potentials between different media and phases. The diffusion of impurities from one medium to another is required to achieve equilibrium [12]. Consequently we studied the distribution of impurities identified to be responsible for KamLAND's low energy background with regards to their behavior in different media and phases. These included:

- vapor-liquid phases in distillation

- solid-liquid phases in adsorption, as well as filtering and isotope exchange

Table 1: Measured radioactivity concentrations in KamLAND LS. The limit for ${ }^{39} \mathrm{Ar}$ was derived from solubility arguments [7] based on air concentration levels [11].

\begin{tabular}{ll}
\hline Isotope & Concentrations $[\mathrm{g} / \mathrm{g}]$ \\
\hline \hline${ }^{39} \mathrm{Ar}$ & $<4.3 \times 10^{-21}$ \\
${ }^{40} \mathrm{~K}$ & $(1.30 \pm 0.11) \times 10^{-16}$ \\
${ }^{85} \mathrm{Kr}$ & $(6.10 \pm 0.14) \times 10^{-20}$ \\
${ }^{210} \mathrm{~Pb}$ & $(2.06 \pm 0.04) \times 10^{-20}$ \\
${ }^{232} \mathrm{Th}$ & $(8.24 \pm 0.49) \times 10^{-17}$ \\
${ }^{238} \mathrm{U}$ & $(1.87 \pm 0.10) \times 10^{-18}$ \\
\hline
\end{tabular}



of this work. analytical problem.
In addition we studied the impact of heating on the lead removal efficiency to target possible organometallic lead compounds formed after alpha decay and known to be prone to ${ }^{124}$ heat-induced break up. This paper reports measured reduction ${ }_{125}$ factors for these different purification methods. Studies done on the removal of radioactive gases $\left({ }^{85} \mathrm{Kr}\right.$ and $\left.{ }^{222} \mathrm{Rn}\right)$ are de- ${ }_{127}$ scribed in [13] and are not covered in this work. Final reduction factors achieved from full-scale scintillator purification in ${ }_{120}$ the KamLAND detector are not within the scope of this paper.

The experimental procedures used throughout this study are ${ }_{131}$ outlined in Sec. 2. In Sec. 3 we discuss the analytical techniques and methods used to quantify and remove radon decay products from LS and its constituents PC, $n$-dodecane, and PPO. Finally, ${ }_{134}$ in Sec. 4 , we discuss the implications and significant findings

\section{Experimental Procedures and Detection Methods}

Measuring the large reductions in ${ }^{210} \mathrm{~Pb}$ needed for Kam-138 LAND's low background phase required the quantitative deter-139 mination of lead concentrations at a level of $10^{-24}$ to $10^{-20} \mathrm{~g} / \mathrm{g} .{ }^{140}$ The starting value corresponds to approximately $4.6 \times 10^{4}{ }^{210} \mathrm{~Pb}^{141}$ atoms per liter of LS, while the smaller concentration represents ${ }^{142}$ the goal for adequately purified LS. Clearly this is a challenging ${ }^{143}$

The measurement of lead concentrations at such extremely ${ }^{145}$ low levels is best achieved using radioactive tracers. The iso- ${ }^{146}$ tope of concern, ${ }^{210} \mathrm{~Pb}$, is a low Q-value beta emitter. Its daugh- ${ }^{147}$ ters, ${ }^{210} \mathrm{Bi}$ and ${ }^{210} \mathrm{Po}$, are pure beta and alpha emitters, respec-148 tively. None of these decays offer a convenient experimental ${ }^{149}$ signature suited to measure small concentrations in lab scale ${ }^{150}$ experiments. Furthermore, ${ }^{210} \mathrm{~Pb}$ has a half life of $22.3 \mathrm{y}$ resulting in a relatively small specific activity of $4.5 \times 10^{-5} \mathrm{~Bq} / \mathrm{L} \mathrm{at}_{15}$ the starting concentration of $10^{-20} \mathrm{~g} / \mathrm{g}$. To overcome this ex- ${ }_{152}$ perimental challenge, we assume that all isotopes of lead born via alpha decay have similar chemical characteristics in organic 153 media, and thus, similar responses to purification techniques.154 Isotope-specific studies showed a factor of 3 higher ${ }^{212} \mathrm{~Pb}$ re-155 duction than observed for ${ }^{214} \mathrm{~Pb}$ [7]. This might be attributed to156 differences in nuclear recoil energies and lead residence times157 in the LS. Given the interest in large reduction factors, and not ${ }^{158}$ analytic precision, the possible impact of these effects are ne-159 glected in this work.

The short lived isotopes of ${ }^{212} \mathrm{~Pb}\left(T_{1 / 2}=10.64 \mathrm{~h}\right)$, born ${ }^{161}$ via ${ }^{216} \mathrm{Po}\left({ }^{220} \mathrm{Rn}\right.$ daughter $)$, and ${ }^{214} \mathrm{~Pb}\left(T_{1 / 2}=26.8 \mathrm{~min}\right)$, born ${ }^{162}$ via ${ }^{218} \mathrm{Po}\left({ }^{222} \mathrm{Rn}\right.$ daughter), can be used to study lead removal ${ }^{163}$ within our target concentration range of $10^{-20}$ to $10^{-24} \mathrm{~g} / \mathrm{g} .164$ At a concentration of $10^{-20} \mathrm{~g} / \mathrm{g}$ the specific ${ }^{212} \mathrm{~Pb}$ activity is 165 $0.83 \mathrm{~Bq} / \mathrm{L}$, a factor $1.8 \times 10^{4}$ larger than that of ${ }^{210} \mathrm{~Pb}$ at equal 166 concentration. Characteristic gamma radiation, of energies 167 $238 \mathrm{keV}$ and $351 \mathrm{keV}$, are emitted after the beta decays of ${ }^{212} \mathrm{~Pb}_{168}$ and ${ }^{214} \mathrm{~Pb}$, respectively. This allows for convenient detection ${ }_{169}$ with a low-background germanium $(\mathrm{Ge})$ detector. The subse-170 quent fast Bi-Po beta-alpha decay sequences following these ${ }_{171}$ lead decays allow the further utilization of delayed coincidence counting with LS for high-sensitivity measurements.

\subsection{Determination of the Radio-nuclide Reduction Factors}

The effectiveness of various lead removal techniques was tested by loading LS with trace amounts of radioactive ${ }^{212} \mathrm{~Pb}$ or ${ }^{214} \mathrm{~Pb}$. This was achieved by first dissolving ${ }^{220} \mathrm{Rn}$ or ${ }^{222} \mathrm{Rn}$, exploiting the excellent solubility of radon in organic solvents, and then allowing it to decay into the meta stable lead isotopes. The dissolved lead activity, $A_{i}$, was measured after loading. The remaining activity, $A_{f}$, was measured after the loaded solution had been subjected to a specific purification procedure. Both activities were corrected to correspond to a common reference time. The reduction factor $R$ for a given species is defined as:

$$
R=\frac{A_{i}}{A_{f}}
$$

Two detector types were used to determine the lead activities. For samples with specific activities of more than $1 \mathrm{~Bq} / \mathrm{L}, \mathrm{Ge}$ detectors were utilized to measure the gamma radiation emitted in ${ }^{214} \mathrm{~Pb},{ }^{214} \mathrm{Bi},{ }^{212} \mathrm{~Pb},{ }^{212} \mathrm{Bi}$ and ${ }^{208} \mathrm{Tl}$ beta decays. For low activity samples, liquid scintillation counting was employed, utilizing the delayed beta-alpha coincidences from the ${ }^{212} \mathrm{Bi}^{212} \mathrm{Po}$ $\left({ }^{220} \mathrm{Rn}\right.$ decay chain) and ${ }^{214} \mathrm{Bi}^{214} \mathrm{Po}\left({ }^{222} \mathrm{Rn}\right.$ decay chain) decay sequences. The latter technique allowed the nearly backgroundfree measurement of specific activities down to $10 \mathrm{mBq} / \mathrm{L}$. Together, these detection techniques provided a means to perform studies of purification reduction factors spanning five orders of magnitude and reaching ${ }^{212} \mathrm{~Pb}$ concentrations as low as $10^{-22} \mathrm{~g} / \mathrm{g}$. Further sensitivity to the target concentration of $10^{-24} \mathrm{~g} / \mathrm{g}$ can only be achieved using the KamLAND detector itself.

\subsection{Scintillator Loading and Purification Procedures for Radon Daughters}

Gas flow-through sources from Pylon Electronics Inc. (models TH-1025 and RN-1025) were used to load LS with ${ }^{220} \mathrm{Rn}$ or ${ }^{222} \mathrm{Rn}$, respectively. A second ${ }^{220} \mathrm{Rn}$ source was made using a $\mathrm{ThO}_{2}$ powder. In all instances $\mathrm{N}_{2}$ was utilized as the carrier gas. To remove dust particles from the gas stream, the $\mathrm{N}_{2}$ was passed through $100 \mu \mathrm{m}$ and $0.8 \mu \mathrm{m}$ filters prior to a flow-through source. The radon-loaded $\mathrm{N}_{2}$ gas was then passed through $500 \mathrm{~cm}^{3}$ of LS via a glass bubbler, fitted with a sparger to break up the gas for increased radon absorption. Flow meters were attached to the input and output gas stream to monitor flow rates.

Our studies showed that the use of a filtered carrier gas was important for achieving reproducible results. Particulates carried by the gas stream readily collect charged radon decay products and are efficiently removed by filtering, thus "faking" a removal mechanism not present in KamLAND scintillator.

Lead removal experiments were performed with LS, ndodecane, PC, and PPO. All samples were handled using the following standardized procedure (unless otherwise noted): 
1. Sparge liquid with radon-loaded $\mathrm{N}_{2}$ for a maximum of $24_{221}$ hours. $^{2}$

2. Transfer the ${ }^{212,214} \mathrm{~Pb}$-loaded liquid into a counting con-223 tainer to determine the initial activity $A_{i}$. For counting with 224 Ge detectors, two $125 \mathrm{~cm}^{3}$ Nalgene ${ }^{\circledR}$ bottles were used.225 If liquid scintillation counting was to be used, half of the 226 sample was transferred to a $10.80 \mathrm{~cm}$ diameter, $4.44 \mathrm{~cm}^{227}$ long acrylic cell, fitted with a $7.62 \mathrm{~cm}$ diameter photo-228 multiplier tube (PMT). The counted LS volume was typi-229 cally $200 \mathrm{~cm}^{3}$.

3. When using the Ge detectors, the counted liquid was trans-231 ferred into the purification system. When using liquid ${ }^{232}$ scintillation counting, sufficient liquid was prepared to al-233 low parallel counting and purification. This method as-234 sumes a homogeneous distribution of lead in the LS.

4. After purification, the liquid was counted using the appro- ${ }^{236}$ priate detector to determine the final activity $A_{f}$.

The "before" and "after" counting was always performed us- ${ }^{239}$ ing the same detector to allow for cancellation of systematic ${ }^{240}$ errors. In some instances it was necessary to use only a single ${ }^{241}$ component of the LS during the purification process. If an $\mathrm{LS}^{242}$ component was analyzed by liquid scintillation counting a scin- ${ }^{243}$ tillating cocktail had to be made prior to loading the counting ${ }^{244}$ cell. This dilution was taken into account in the reported re- ${ }^{245}$ duction factor. Furthermore, measurements were carried out to ${ }^{246}$ cross calibrate the two different detector types.

Detailed studies of systematic errors were performed on ev- ${ }^{24}$ ery aspect of the purification processes. Thorough cleaning of ${ }^{249}$ all containers used in the loading and counting was impera- ${ }^{250}$ tive when dealing with extremely low levels of radioactivity. ${ }^{25}$ Cross contamination had to be avoided in order to achieve re- ${ }^{252}$ producible results. When chemically compatible, all components were cleaned with high purity solvents (analytic grade or better). Each part was cleaned with acetone, then with 1 molar ${ }^{254}$ $\mathrm{HNO}_{3}$, followed by a rinsing with de-ionized (DI) water and ${ }_{256}^{255}$ ethanol.

In the case of Ge detector counting, it was found that a sub- ${ }^{257}$ stantial fraction (up to $50 \%$ ) of the lead activity contained in ${ }^{258}$ the LS could adhere to the walls of the Nalgene bottles used ${ }^{259}$ for the counting. The bottles were thus counted again after the ${ }^{260}$ lead loaded scintillator had been transferred into the purifica- ${ }^{261}$ tion setup to account for this loss mechanism.

\section{Removal of Radon-Born Lead from Liquid Scintillator}

In any purification process, the difference in concentrations ${ }_{267}^{266}$ and solubilities between phases drives the separation of impu- ${ }_{268}$ rities between them. In this work these phases were realized in ${ }_{269}$ the form of water-oil and silica-oil systems or gas-liquid phases. ${ }_{270}$ Processes which rely primarily on diffusion of the impurities between phases require initial concentrations in the transient

${ }^{2}$ Care must be taken when loading LS or pure PC. PC has a high vapor ${ }^{274}$ pressure causing it to evaporate during bubbling, resulting in a lowering of the $\mathrm{e}^{275}$ PC concentration as well as corrosion of filters and membranes. phase to be less than those listed in Table 1. The mass transfer involved in such diffusive processes can be modeled assuming thermodynamic equilibrium. The work presented here does not report the reduction in terms of a Gibbs potential, as done in previous publications [14]. Instead, we report the results in the form of a reduction factor as defined in Eq. 1. We found this to be sufficient for the purpose of identifying the most effective method of purification.

The following purification techniques were studied: water extraction, ion enhanced water extraction, isotope exchange, filtering, adsorption and distillation. When applicable, experiments were performed in series with one another to establish if the methods scale as the product of their individual reduction factors. For each of these techniques, the reduction factor, time of operation, solute volume, and initial activity were studied for correlations.

Among the ${ }^{222} \mathrm{Rn}$ daughters ${ }^{210} \mathrm{~Pb}$ is of particular interest because of its long half life. Following the assumption discussed in Section 2 that different alpha-born isotopes of lead in organic media have similar characteristics, the purification techniques described below were evaluated using ${ }^{212} \mathrm{~Pb}$ and ${ }^{214} \mathrm{~Pb}$ as tracers. However, one cannot assume that all metallic radon daughters are removed with equal efficiency as they have different chemical characteristics. The radionuclides ${ }^{208} \mathrm{Tl},{ }^{212} \mathrm{Bi}$, ${ }^{214} \mathrm{Bi}$ and ${ }^{218} \mathrm{Po}$ were analyzed separately to better understand the variation in the reduction factors across chemical species. For example, experimental information obtained on ${ }^{218}$ Po was used to gain some insight into how much ${ }^{210} \mathrm{Po}\left(T_{1 / 2}=138 \mathrm{~d}\right)$ would remain in the LS after purification. The results of these non-lead purification studies are not reported here. More details on the purification research performed with KamLAND LS can be found in $[7,13,15-21]$.

\subsection{Water Extraction}

A water purification system already existed in KamLAND; it had been used during the initial filling [22]. We thus first tried to understand whether this system could be used, perhaps with modification, to remove dissolved lead from KamLAND LS. A miniature water extraction system was built to circulate LS through a glass purification column filled with DI water, obtained from a Millipore Direct-Q5 (18.2 M $2 \cdot \mathrm{cm}$ resistivity) water purifier. The lead content of the purified water was not determined. The LS, which is less dense than water, was removed from the top of the water column and re-circulated back to the bottom of the column using a peristaltic pump. To increase the surface to volume ratio, the LS was broken into small bubbles with a custom-made glass frit affixed to the bottom of the column.

Water extraction experiments showed weak lead reduction factors between 1.02 and 1.10 . Using about $500 \mathrm{~cm}^{3}$ of water, $250 \mathrm{~cm}^{3}$ of LS and a flow rate of $200 \mathrm{~cm}^{3} / \mathrm{min}$, the observed reduction factors saturated for circulation times longer than 5 hours [7]. A second set of independent experiments was performed using $50 \mathrm{~cm}^{3}$ of lead-loaded LS with $60 \mathrm{~cm}^{3}$ of distilled water mixed with 0.01 to $6 \mathrm{~mol} / \mathrm{L}$ of $\mathrm{HNO}_{3}, \mathrm{HCl}, \mathrm{NaOH}$ [16]. Mixing was achieved by vigorously shaking for $5 \mathrm{~min}$. In these experiments we observed saturation levels consistent with the 


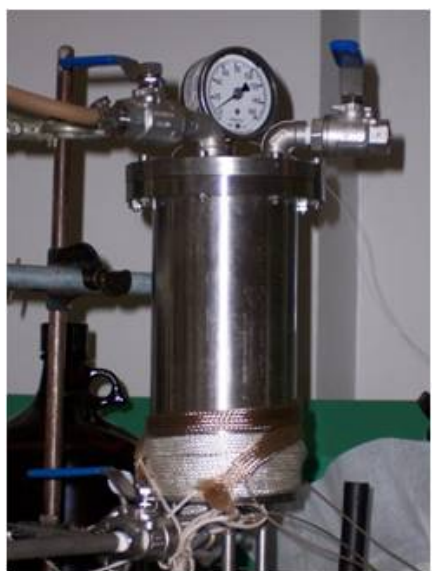

(a)

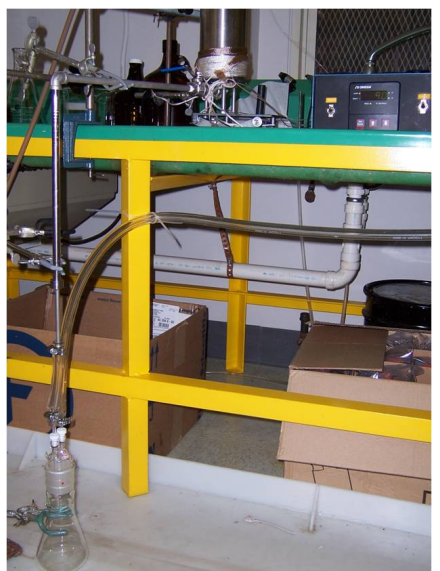

(b)

Figure 2: The stainless steel vessel used to heat the LS prior to adsorption is ${ }_{310}$ depicted in Fig. 2(a). Fig. 2(b) is the adsorption column in which all silica gel experiments were performed. At the top one can see the heating vessel and ${ }^{311}$ thermocouple. Furthermore, a vacuum line was used to regulate the flow rate ${ }^{312}$ through the silica column.

more controlled mixing noted above. In all experiments the lead reduction factor showed a clear decrease with growing $\mathrm{pH}_{318}^{317}$ However, the magnitude of this change over the $\mathrm{pH}$ range never ${ }_{319}$ exceeded $30 \%$. Therefore, water extraction was found to be not well suited for achieving the very large lead reduction factors needed in KamLAND.

\subsection{Isotope Exchange, Filtration, and HTiO Adsorption}

LS loaded with radioactive lead was circulated through a bed of granular lead, or lead shot. Assuming that the dissolved lead ${ }_{325}$ forms an equilibrium with the essentially infinite number of sta- ${ }_{326}$ ble lead atoms, we tested the possibility of isotope exchange ${ }_{327}$ These experiments showed no quantifiable lead reduction ef- ${ }_{328}$ fect beyond the measured loss to the surfaces of the pumping ${ }_{329}$ system [7].

Early studies showed that filtering loaded LS removed the dissolved lead in a variable manner. This fluctuation in $\operatorname{lead}_{332}$ removal efficiency was traced to the presence of dust parti- ${ }_{33}$ cles in the carrier gas stream to which the charged radon decay ${ }_{33}$ products readily attach [23]. Carefully filtering the gas stream 335 avoided this interference and enhanced the reproducibility of ${ }_{336}$ the experiments.

HTiO has successfully been used in the SNO experiment toз38 remove actinides from light and heavy water [24]. We investi-3з9 gated this method for its suitability in removing lead from or-340 ganic LS. HTiO pre-loaded HEPA PTFE filters (surface density 341 $0.64 \mathrm{~g} / \mathrm{cm}^{2}$ ), supplied by the Oxford University SNO group,, 342 were utilized for these tests. lead-loaded LS was placed into 343 a $60 \mathrm{ml}$ syringe attached to the filter and the liquid was pushed ${ }_{344}$ slowly through the filter. The filter was replaced after every $125_{345}$ $\mathrm{ml}$ of lead-loaded LS. For flow rates of 3.3 and $16.5 \mathrm{~cm}^{3} / \mathrm{min} \mathrm{we}_{346}$ observed lead reduction factors of $1.06 \pm 0.01$ and $1.07 \pm 0.01,347$ respectively. No further consideration was given to this method. 348
Table 2: ${ }^{212} \mathrm{~Pb}$ reduction factors obtained with $250 \mathrm{ml}$ of LS utilizing different purification modes. The uncertainties given for the Selecto results are statistical plus systematic added in quadrature. The Aerosil results have only statistical uncertainties. Tests with Selecto used $10 \mathrm{~g}$ of silica while only $0.5 \mathrm{~g}$ of Aerosil was used.

\begin{tabular}{llc}
\hline \multicolumn{1}{c}{ Adsorbent } & \multicolumn{2}{c}{ Reduction Factor } \\
& \multicolumn{1}{c}{ Batch } & Loop \\
\hline \hline $32-63 \mu$ m Selecto & $22.73 \pm 0.52$ & $4.98 \pm 0.12$ \\
$100-200 \mu$ m Selecto & $15.38 \pm 0.24$ & $7.04 \pm 0.10$ \\
Aerosil 200 & $3.45 \pm 0.02$ & $1.49 \pm 0.02$ \\
\hline
\end{tabular}

\subsection{Chromatography with Silica Gels (Adsorption)}

Previous studies identified silica gel extraction as an efficient means of removing inorganic impurities from LS [14, 25-27]. This method was studied in depth to evaluate its suitability for removing lead using different adsorption materials packed in chromatographic columns. Plate theory [28] is commonly used to describe liquid-solid chromatography, which requires mass and thermodynamic equilibrium between the mobile (LS) and stationary (silica gel) phases. One can divide the column into many distinct sections, referred to as plates. The concentration of an impurity in successive plates can be derived from mass balance and equilibrium conditions:

$$
\mathrm{X}_{\mathrm{M}(\mathrm{p})}=\frac{\mathrm{X}_{0} \nu^{\mathrm{p}}}{\mathrm{p} !} \mathrm{e}^{-\nu}, \quad \mathrm{p}=1,2,3, \ldots
$$

where $\mathrm{X}_{0}$ is the initial concentration of the impurity, $\mathrm{X}_{\mathrm{M}(\mathrm{p})}$ is the concentration in the $\mathrm{p}^{\text {th }}$ plate, and $v$ is the plate volume. Eq. 2 is referred to as the elution curve and indicates that given enough adsorbent, there is no theoretical limit to the achievable reduction factor.

There are two possible methods for purifying LS with this technique. One can assume that the silica gel is infinitely adsorptive and run the liquid through a column in a loop mode, or make a single pass through the column in a batch mode. Experiments showed batch purification through a long column to be the method of choice, as multiple passes through the same column lead to lower reduction factors. Batch mode makes efficient use of the concentration gradient of the solute developing along the column. Our observation that batch mode provides higher purification factors than loop mode agrees well with previous studies [14]. To illustrate this point, Table 2 lists the lead reduction factors obtained for three different types of silica gel used in both batch and loop mode.

All results presented from here on are based on batch mode operation. A wide variety of silica gels are commercially available. For this study a $32-63 \mu \mathrm{m}$ (grain size) silica gel from Selecto Scientific was used as the standard. Our laboratory chromatographic lead purification system, shown in Fig. 2(b), was designed and operated in the following way: a slightly pressurized stainless steel reservoir, holding approximately $10 \mathrm{~L}$ of LS, was coupled to a $35 \mathrm{~cm}$ long stainless steel chromatographic column with $0.97 \mathrm{~cm}$ inner diameter. Valves installed in the LS 
path allowed flow regulation through the column. The column ${ }_{405}$ was fitted with a $10 \mu \mathrm{m}$ stainless steel mesh filter at the outlet 400 to sequester the adsorbent.

Prior to runs with LS, the silica gel was first conditioned ${ }^{407}$ (compacted). This was done to keep eddies and voids from ${ }^{408}$ developing inside the column. Sufficient compactification was achieved by providing constant $\mathrm{N}_{2}$ pressure to the top of the col- $^{409}$ umn while pulling partial vacuum at the bottom (without LS). ${ }^{410}$ Our standard purification procedure gave consistent and repro- ${ }^{41}$ ducible lead removal factors, as verified by repeated measure ${ }^{412}$ ments with the standard gel. The procedure consisted of the ${ }^{413}$ following steps:

1. Clean all parts with acetone, then with $0.1-1.0$ molar 415 $\mathrm{HNO}_{3}$, followed by a rinse with DI water and ethanol.

2. Add the desired amount of silica gel to the column, close $^{416}$ all valves, and pump down to $100 \mathrm{hPa}$. Assist setting of ${ }^{417}$ the silica granules by knocking the column during pump- ${ }^{418}$ down.

3. Apply $1000 \mathrm{hPa} \mathrm{N}$ pressure to the top of the column and $\mathrm{d}^{420}$ continue to knock the column to set the silica in place. The ${ }^{42}$ pressure at the outlet rises to approximately $300 \mathrm{hPa}$ when ${ }^{422}$ equilibrium is reached.

4. Remove the vacuum pump and slowly release the pressure ${ }^{424}$ from the inlet side of the system.

5. Add the lead-loaded LS to the reservoir with all valves ${ }^{426}$ closed. Pressurize the reservoir with $\mathrm{N}_{2}$ to $1000 \mathrm{hPa}$.

6. Slowly open the valve between the reservoir and the col- $^{42}$ umn to wet the gel. Controlled release of LS prevents $\operatorname{cav}_{-}{ }^{429}$ ities from forming at the top of the silica column. The flow ${ }^{430}$ rate of LS through the column was $50 \mathrm{ml} / \mathrm{min}$.

7. After purification, the $\mathrm{LS}$ is passed through a $0.2 \mu \mathrm{m} \mathrm{PTFE}{ }^{432}$ HEPA filter to remove silica particulates suspended in the ${ }^{433}$ LS.

Further conditioning tests were performed by heating the sil-436 ica gel to $100-120^{\circ} \mathrm{C}$ to remove adsorbed water from its sur-437 face. Water molecules are known to adhere to the adsorption ${ }_{438}$ sites and reduce the effectiveness of the silica gel [28]. How-439 ever, this conditioning was not found to provide any benefit in 440 terms of boosting lead removal.

Using the procedure outlined above, lead removal studies 442 were performed with many different types of adsorbents. The 443 results of these experiments are given in Table 3. Our procedure 444 provided reproducible results, and typical statistical uncertain-445 ties on individual runs were of order $0.1 \%$. Repeated runs with 446 the $32-63 \mu \mathrm{m}$ silica gel yielded a standard deviation of $7.2 \%$ for the lead reduction factor, which we interpret as a measure 448 of the systematic uncertainty.

Since, as already mentioned, the elution curve (Eq. 2) indi-450 cates that in principle the reduction factor has no limit, making ${ }_{451}$ the chromatographic column arbitrarily long should allow one 452 to achieve any desired reduction factor. This behavior was not 453 observed, as a maximal reduction factor of 29 was achieved 454 for a single pass purification. While this factor is substantial,455 it falls short of the required value for KamLAND, so studies456 were performed to determine whether this limitation could be 457 overcome.
We considered three hypotheses to explain the observed limit in the lead reduction factor during silica gel extraction:

Hypothesis 1. There exists lead cross-contamination between runs (insufficient cleaning).

Hypothesis 2. The intrinsic uranium and thorium concentrations of the silica gel, and the resulting out-gassing radon, reintroduces ${ }^{212} \mathrm{~Pb}$ into the $\mathrm{LS}$. The observed limit may be determined by the equilibrium between the removal and addition of $P b$.

Hypothesis 3. Some fraction of the ${ }^{212} \mathrm{~Pb}$ may exist in the $L S$ in a form without affinity towards the silica gel.

Blank runs performed with unloaded LS following a purification run with lead-loaded LS did not show any carry-over activities, thus excluding Hypothesis 1 and constraining also Hypothesis 2. Furthermore, bottles and containers were not reused whenever possible, and all re-used equipment underwent vigorous cleaning, as described in Sec. 2.2.

Hypothesis 2 was further refuted by measuring the radioactive content of the gel. The $32-63 \mu \mathrm{m}$ Selecto Scientific silica gel was measured to have an intrinsic uranium and thorium content of $167 \mathrm{ppb}$ and $152 \mathrm{ppb}$, respectively [30], resulting in $1.7 \mathrm{mBq}$ of ${ }^{212} \mathrm{~Pb}$ in $2.5 \mathrm{~g}$ of silica gel. Typical loading activities for the LS were $5 \mathrm{kBq}$. This silica gel had an average reduction factor of 19 , resulting in $250 \mathrm{~Bq}$ of residual ${ }^{212} \mathrm{~Pb}$ activity. The total intrinsic activity of the silica gel is thus much smaller than the measured activity after silica gel purification, implying that the former contributes negligibly to the observed limit in the reduction factor.

Hypothesis 3 was also tested experimentally. Chromatographic impurity removal techniques selectively address polar molecules or ionic states [28,31]. It is the excess charge on the molecule, or atom, which draws it to the hydroxyl group $(-\mathrm{OH})$ on the active adsorption sites of the gel. The excellent reproducibility of our lead removal experiments suggests that about $3-5 \%$ of the lead atoms were present in a non-polar, molecular state, without affinity to the active hydroxyl groups of silica gel.

In order to understand why this species has no affinity to the silica gel, it is of particular interest to examine a study performed with $n$-dodecane exposed to a ${ }^{60} \mathrm{Co}$ source [32]. This study showed that the exposure to ionizing radiation produced fragmented carbon chains. We note that the ${ }^{212} \mathrm{~Pb}$ and ${ }^{214} \mathrm{~Pb}$ isotopes used in our studies, as well as the ${ }^{210} \mathrm{~Pb}$ present in KamLAND's LS, are born via alpha decay. A recoiling lead nucleus, with typical energies of a few hundred $\mathrm{keV}$, can easily fragment the carbon-carbon bonds in $n$-dodecane, having typical binding energies of order $10 \mathrm{eV}$ [29]. These fragmented molecular states could form stable, electrically neutral, non-polar compounds. The most notable of these are the lead-carbon bonds which tend to be covalent, and thus non-polar. The decay of the parent nuclei into lead occurs in a carbon rich environment and this bonding is energetically favored over many other available chemical bonds (e.g. C-C bonds) [29]. The non-polar leadcarbon compounds would have no affinity to the polar reaction sites on silica gel, supporting Hypothesis 3. 
Table 3: Measured ${ }^{212} \mathrm{~Pb}$ reduction factors in LS. Only statistical uncertainties are quoted. Experimental investigation of the systematic uncertainty for $32-64 \mu \mathrm{m}$ gel yielded $7.2 \%$. The uncertainty on the mass measurements was $0.05 \mathrm{~g}$.

\begin{tabular}{lll}
\hline Adsorbent Type & lead Reduction Factor & Adsorbent Mass [g] \\
\hline \hline Selecto, Lot \#301286301, Si-gel 32-63 $\mu \mathrm{m}$ & $19.4 \pm 0.47$ & 5.0 \\
Selecto, Lot \#306279301, Si-gel 100-200 $\mu \mathrm{m}$ & $15.38 \pm 0.24$ & 10 \\
Selecto, Lot \#102085402, Alusil 70 & $27.03 \pm 0.73$ & 5.0 \\
Selecto, Lot \#109110402, Alusil Plus & $8.33 \pm 0.07$ & 2.0 \\
Selecto, Lot \#108110403, Alusil NanoSmart & $8.20 \pm 0.07$ & 2.0 \\
Selecto, Lot \#900110401, Si-gel NanoSmart ACT & $3.58 \pm 0.01$ & 2.0 \\
Selecto, Lot \#900110401, Alusil Coarse & $28.57 \pm 0.82$ & 5.0 \\
Selecto, Lot \#107223405, Alusil 40 without K & $10.31 \pm 0.21$ & 2.0 \\
Aldrich, 3-(Diethylenetriamino) & $8.33 \pm 0.69$ & 10.0 \\
Propyl-Functionalized gel & & 10.2 \\
Aldrich, Triamine Tetraacetate-Functionalized gel & $11.11 \pm 0.12$ & 0.5 \\
Aerosil 200 & $8.26 \pm 0.07$ & 15 \\
Süd-Chemie, Cu/Mn Catalyst T-2550 & $3.45 \pm 0.02$ & 7.2 \\
Süd-Chemie, Cu/Mn Catalyst T-2550, Crushed & $26.32 \pm 0.69$ & 2.0 \\
Ca $(\text { PO } 4)_{2}$ & $6.54 \pm 6.54$ & \\
\hline
\end{tabular}

Table 4: Chemical characteristics of the LS components and of some known organometallic lead compounds. These data are taken from the CRC [29].

\begin{tabular}{llcc}
\hline Name & Formula & Density [g/cm ${ }^{3}$ ] @ 20 ${ }^{\circ} \mathrm{C}$ & Boiling Point [ ${ }^{\circ} \mathrm{C}$ ] \\
\hline \hline n-Dodecane & $\mathrm{C}_{12} \mathrm{H}_{26}$ & 0.7495 & 215 \\
1,2,4-Trimethylbenzene (PC) & $\mathrm{C}_{9} \mathrm{H}_{12}$ & 0.8758 & 169 \\
2,5 Diphenyloxazole (PPO) & $\mathrm{C}_{15} \mathrm{H}_{11} \mathrm{NO}$ & 1.0940 & $360^{1}$ \\
Tetraethyllead & $\mathrm{C}_{8} \mathrm{H}_{20} \mathrm{~Pb}$ & 1.653 & $200^{2}$ \\
Methyltriethyllead & $\mathrm{C}_{7} \mathrm{H}_{18} \mathrm{~Pb}$ & 1.71 & 70 \\
Diethyldimethyllead & $\mathrm{C}_{6} \mathrm{H}_{16} \mathrm{~Pb}$ & 1.79 & 51 \\
Ehtyltrimethyllead & $\mathrm{C}_{5} \mathrm{H}_{14} \mathrm{~Pb}$ & 1.88 & 27 \\
Tetramethyllead & $\mathrm{C}_{4} \mathrm{H}_{12} \mathrm{~Pb}$ & 1.995 & 110 \\
\hline
\end{tabular}

${ }^{1} \mathrm{PPO}$ has a melting point of $74^{\circ} \mathrm{C}$

${ }^{2}$ Tetraethyllead will decompose at $200{ }^{\circ} \mathrm{C}$ before it boils.

Table 4 lists the relevant properties of the known organic ${ }_{471}$ compounds containing lead. All exhibit lower boiling points 472 than those of PC and $n$-dodecane. It is of interest to note 473 $\mathrm{C}_{8} \mathrm{H}_{20} \mathrm{~Pb}$ is thermally unstable and will decompose at $200{ }^{\circ} \mathrm{C} .474$ This process would leave behind a polar or ionic lead species. 475

To obtain supporting evidence for Hypothesis 3, we exper- ${ }^{476}$ imented with chemical procedures targeting the organic lead- ${ }^{477}$ carbon bonds. It is known that the reagents: $\mathrm{FeCl}_{3}[33],{ }^{478}$ $\mathrm{SnCl}_{4}$ [34], $\mathrm{MoS}_{2}$ [35] and thiol resin [36] target these lead- ${ }^{479}$ carbon bonds directly.

To test the effect of these chemicals on the lead reduction factor, we first passed ${ }^{212} \mathrm{~Pb}$-loaded LS through a silica gel col- umn to remove the ionic lead component. In a second step, $150 \mathrm{ml}$ of the treated LS was stirred magnetically, with one of the reagents listed above, for $45 \mathrm{~min}$. Next, $15 \mathrm{ml}$ of $0.5 \mathrm{M}$ EDTA $\left(\left[\mathrm{CH}_{2} \mathrm{~N}\left(\mathrm{CH}_{2} \mathrm{CO}_{2} \mathrm{H}\right)_{2}\right]_{2}\right.$ ) solution ( $\mathrm{pH}$ of 8 ) was added to the mixture and stirred for an additional $15 \mathrm{~min}$. The mixture was filtered under partial vacuum and passed over a molecular sieve to remove any water. The resulting LS was filtered a second time to remove any particulates of the molecular sieve. The liquid was counted to determine the remaining amount of ${ }^{212} \mathrm{~Pb}$. The lead reduction factors obtained this way are tabulated in Table 5. 
Table 5: Combined ${ }^{212} \mathrm{~Pb}$ reduction factors for purification methods performed in series with an initial $\mathrm{SiO}_{2}$ purification. Secondary techniques specifically target lead-carbon bonds. Only statistical uncertainties are reported except where noted.

\begin{tabular}{lc}
\hline \multicolumn{1}{c}{ Method } & Reduction Factor \\
\hline \hline $\mathrm{SiO}_{2}$ & $19.4 \pm 0.47^{1}$ \\
$\mathrm{SiO}_{2} \rightarrow \mathrm{FeCl}_{3}$ & $>1060$ \\
$\mathrm{SiO}_{2} \rightarrow \mathrm{FeCl}_{3} \rightarrow \mathrm{SiO}_{2}$ & $>1263$ \\
$\mathrm{SiO}_{2} \rightarrow \mathrm{SnCl}_{4}$ & $47.0 \pm 1.1$ \\
$\mathrm{SiO}_{2} \rightarrow \mathrm{MoS}_{2}$ & $24.9 \pm 0.6$ \\
$\mathrm{SiO}_{2} \rightarrow \mathrm{ThiolResin}$ & $22.7 \pm 0.5$ \\
$\mathrm{SiO}_{2} \rightarrow$ Distillation & $>4389^{2}$ \\
\hline
\end{tabular}

${ }^{1}$ Includes Systematic Uncertainties.

${ }^{2}$ Experiment performed with n-Dodecane.

LS treated with $\mathrm{FeCl}_{3}$ before silica gel extraction showed a

50-fold enhanced lead reduction factor compared to plain chromatography. We interpret this boost in effectiveness as supporting evidence for Hypothesis 3. However, the $\mathrm{FeCl}_{3}$ treatment of the LS was found to permanently degrade its optical properties. ${ }^{515}$ The measured light attenuation length at $436 \mathrm{~nm}$ was $<10^{-4}{ }_{516}$ $\mathrm{m}$ compared to approximately $13 \mathrm{~m}$ for untreated LS. Optical $\mathrm{l}_{517}$ transparency could not be restored by means of filtration or re- ${ }_{518}$ peated treatment with silica gel.

The successful experiments utilizing reagents targeting lead- ${ }^{520}$ carbon bonds prompted us to consider heating the lead-loaded ${ }^{521}$ LS to $100-200{ }^{\circ} \mathrm{C}$ to remove the tetraethyllead which decom- ${ }^{522}$ poses [29]. As a test, ${ }^{212} \mathrm{~Pb}$-loaded LS was pre-heated in $\mathrm{a}^{523}$ stainless steel vessel, as shown in Fig. 2(a), before sending it ${ }^{524}$ through the ion-exchange column, depicted in Fig. 2(b). The ${ }^{525}$ results from these heating experiments are provided in Table $6 .^{526}$ The first three entries show a substantial enhancement of the ${ }^{527}$ lead reduction factor for pre-heated LS compared to the exper- ${ }^{528}$ iments with silica gel extraction only. The following three en- ${ }^{529}$ tries give a factor 5 to 10 reduction obtained by just heating the ${ }^{530}$ LS and not using silica gel. Lead adherence to the container ${ }^{531}$ walls for unheated liquid scintillator was excluded by experi- ${ }^{532}$ ments performed at room temperature. The observed lead loss ${ }^{533}$ is therefore heat related and not a property of the scintillator ${ }^{534}$ handling system. The lead loss mechanism was not determined ${ }^{535}$ in this work.

We consider these results to be further evidence for Hypoth-538 esis 3. However, the data are not specific enough as to allow US539 to identify which compounds are present and in what propor-540 tion. This is nevertheless an important finding, indicating that ${ }_{541}$ the limitless reduction factors predicted by plate theory, Eq. 2,542 are not to be expected for alpha decay products in an organic ${ }_{543}$ medium.
Table 6: ${ }^{212} \mathrm{~Pb}$ reduction factors observed for heated LS. All heating was performed in a stainless steel container. The first column gives the heating time. The second column states the average temperature during heating. The final column provides the observed reduction factor.

\begin{tabular}{ccc}
\hline Time [hrs] & Avg. Temp $\left[{ }^{\circ} \mathrm{C}\right]$ & Reduction Factor \\
\hline \hline \multicolumn{3}{c}{ Heating + Silica Gel } \\
2 & 142 & $277.8 \pm 23.2^{1}$ \\
2 & 187 & $200 \pm 16^{1}$ \\
1.5 & 160 & $172.4 \pm 2.97^{2}$ \\
\hline & Heating Only \\
2 & 187 & $5.3 \pm 0.06$ \\
8 & 157 & $5.08 \pm 0.05$ \\
\hline
\end{tabular}

Control Sample, No Heat, Vessel Blank

$3 \quad 17$

$1.11 \pm 0.01$

$15 \mathrm{~g} \mathrm{SiO}_{2}$ in column.

$25 \mathrm{~g}$ of $\mathrm{SiO}_{2}$ and silica gel heated in column.

\subsection{Distillation}

The final method studied for KamLAND's LS purification addresses the removal of all lead species in an integrated way: distillation. During distillation, the distillate is heated to induce a phase change. Differences in vapor pressure result in fractionation between the constituents. As a by-product, the heating further breaks organo-metallic bonds and transforms organometallic lead compounds, that may be in solution, into a polar or ionic form. These, in turn, can be effectively removed by distillation.

Distillation experiments primarily utilized lead-loaded $n$ dodecane, which makes up $80 \%$ of the KamLAND LS. The distillation apparatuses utilized in these studies are pictured in Fig. 3. The system in Fig. 3(a) consisted of a three-neck flask $(500 \mathrm{ml})$, a silvered fractionating column packed with stainless steel wool, and a condenser. A thermocouple was used to regulate the distillate temperature to within a few ${ }^{\circ} \mathrm{C}$. A mercury thermometer was placed on top of the fractionation column to monitor the temperature of the vapor prior to condensation. The stainless steel wool increased the effective number of plates. In essence, it allows for multiple distillations of the liquid as the vapor rises through the column and re-condenses on the steel wool. Typical operating conditions were $170 \mathrm{hPa}$, with a $160 \pm 1{ }^{\circ} \mathrm{C}$ set-point for the distillate in the boiling flask. The flask temperature was maintained by the thermocouple but the temperature measured by the mercury thermometer at the top of the fractionating column varied over time from $125-145^{\circ} \mathrm{C}$. Temperatures exceeding $145^{\circ} \mathrm{C}$ the top of the column resulted in a lower reduction factor due to the boil-over of contaminants. The rate of distillation at these operating conditions was 10 


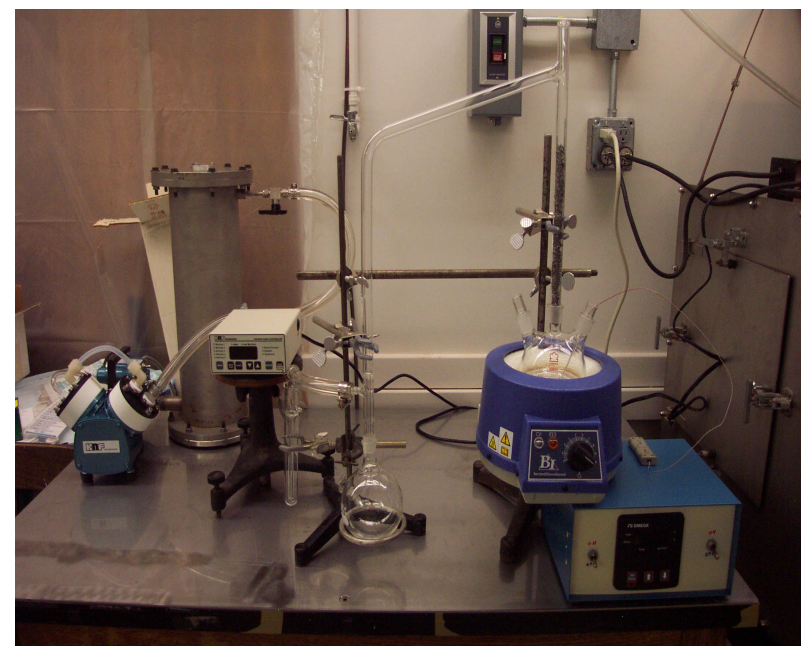

(a)

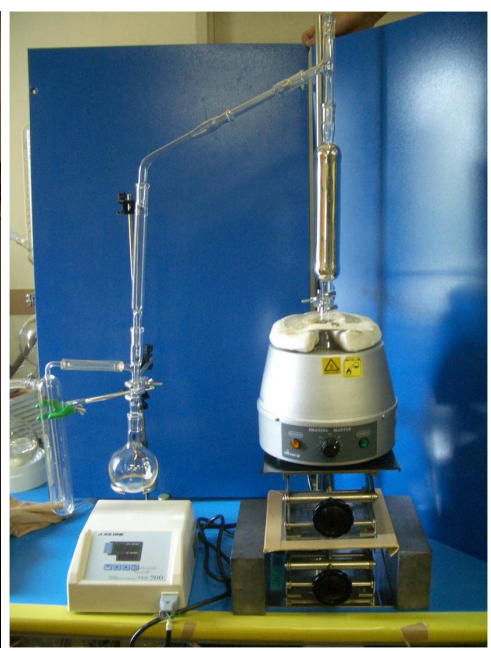

(b)

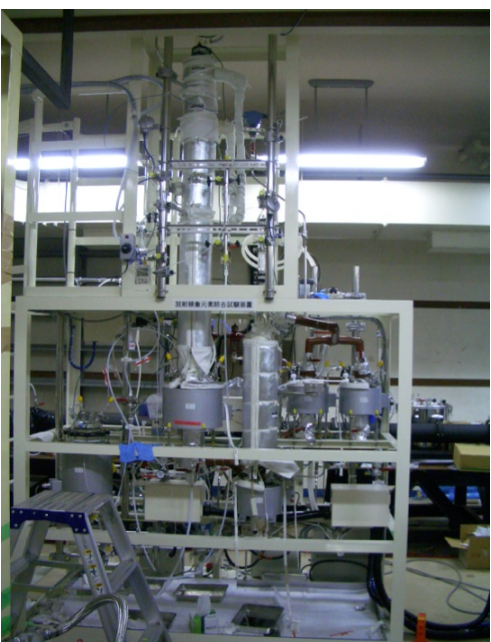

(c)

Figure 3: Size evolution of distillation systems utilized during the purification experiments. Fig. 3(a) and Fig. 3(b) show the bench-top systems used at the University of Alabama and Tohoku University, respectively. Together they provided a complete analysis of systematic studies on distillation. Fig. 3(c) is the scaled-up distillation system at Tohoku University, allowing us to increase the amount of distillate by a factor 20 .

Table 7: Average ${ }^{212} \mathrm{~Pb}$ reduction factors obtained by distillation of various components of LS. The uncertainty is reported as the addition in quadrature of the error on the weighted mean with the standard deviation of the measurements to account for large measurement-to-measurement variations relative to the individual measurement uncertainties (typically $10 \%-20 \%$ )

\section{Distilled material}

n-Dodecane

1 time distillation

3 times distillation

5 times distillation

7 times distillation

$n$-Dodecane

1 time distillation

5 times distillation

5 times distillation $+\mathrm{SiO}_{2}$

n-Dodecane

1 time distillation

3 times distillation

5 times distillation

\section{Pseudocumene}

1 time distillation

2 times distillation

3 times distillation

4 times distillation

PPO

1 time distillation

\section{Averaged Reduction Factors} 300 hPa, $160{ }^{\circ} \mathrm{C}$, Bench-top System $[13,19]$
$(3.4 \pm 2.3) \times 10^{3}$
$(1.9 \pm 1.0) \times 10^{4}$
$(2.2 \pm 0.6) \times 10^{4}$
$(2.8 \pm 0.6) \times 10^{4}$

$190 \mathrm{hPa}, 160{ }^{\circ} \mathrm{C}$, Bench-top System

$(7.8 \pm 3.2) \times 10^{2}$
$(2.6 \pm 0.2) \times 10^{3}$
$(2.9 \pm 0.6) \times 10^{3}$

37 hPa, $113{ }^{\circ} \mathrm{C}$, Scaled-up System [13]

$$
(1.4 \pm 0.3) \times 10^{3}
$$$$
(3.8 \pm 1.2) \times 10^{3}
$$

$$
>1.3 \times 10^{4}
$$

37 hPa, $75^{\circ} \mathrm{C}$, Scaled-up System [18]

$(7.7 \pm 3.2) \times 10^{2}$

$(1.3 \pm 1.2) \times 10^{3}$

$(2.8 \pm 1.3) \times 10^{3}$

$(7.8 \pm 4.8) \times 10^{3}$

$20 \mathrm{hPa}, 160{ }^{\circ} \mathrm{C}$, Bench-top System [19] 
$\mathrm{ml} / \mathrm{min}$. Operating the distillation system at a reduced pressure ${ }_{597}$ is important to minimize the amount of dissolved radioactive 598 gases $\left({ }^{222} \mathrm{Rn},{ }^{85} \mathrm{Kr}\right.$, and $\left.{ }^{39} \mathrm{Ar}\right)$ as well as heat-induced changes599 in the optical properties of the distillate.

Repeated distillation tests with ${ }^{212} \mathrm{~Pb}$-loaded $n$-dodecane pro-601 vided a handle on the variance associated with maintaining con-602 sistent operating procedures. To maintain reproducibility of the 603 distillation results, the following procedures were utilized: 604

- All internal surfaces of the distillation system were ${ }^{605}$ cleaned with acetone, then etched with $0.1-1.0$ molar $^{606}$ $\mathrm{HNO}_{3}$, and rinsed with DI water and ethanol. This elimi- ${ }^{607}$ nated carry-over between subsequent distillations.

- All distillate collected before the top thermometer reached610 the boiling point of the distillate was discarded. This re-611 moves all impurities with a low vapor pressure. Some leador candidates, addressed in this way, can be found in Table 4.613

- Collection of the distillate was stopped after approxi- ${ }_{615}$ mately $90 \%$ of the volume was distilled. This operation reduces boil-over effects and re-contamination with high- ${ }_{617}$ boiling-point compounds.

- A constant rate of distillation was maintained. Rapid boil-619 ing at increased distillation rates directly contributes to the transfer of impurities into the distillate.

Distillation was performed under different pressure and tem-621 perature conditions for $n$-dodecane, PC, and PPO. Similar mea-622 surements taken with the different distillation systems depicted ${ }_{623}$ in Fig. 3 allowed for the investigation of instrumental varia-624 tions in the procedure that could impact the reduction factor.625 To ensure that the reduction factors observed in the modestly626 sized table-top experiments would apply to larger volumes, a627 scaled-up distillation system, shown in Fig. 3(c), was designed628 to process $5 \mathrm{~L}$ at a time. Up to seven distillations were repeated ${ }_{62}$ in series to assess saturation of the lead reduction factor. Distillate samples were taken after consecutive distillations during a single experiment. The measured ${ }^{212} \mathrm{~Pb}$ reduction factors are ${ }^{630}$ summarized in Table 7. Beyond three successive distillations, no further lead reduction could be observed. We also tested the ${ }_{632}^{631}$ combined effect of silica gel extraction and 5-times distillation ${ }_{633}$ resulting in a factor 1.1 improvement compared to distillation6з4 only, as can be seen in Table 7.

Distillation proved to be the most effective means of re ${ }^{636}$ moving radioactive lead from the KamLAND LS components. ${ }_{638}$ The reduction factors observed during the study of distillation 639 ranged from a few hundred to several thousand, depending on ${ }^{640}$ the operating conditions. These results provided the necessary ${ }_{642}^{641}$ technical base required to achieve the high reduction factors ${ }_{643}$ needed for the KamLAND purification campaign. They further644 provided essential procedural development and initial parame ${ }_{646}^{-645}$ ters for the full-scale KamLAND distillation system.

\section{Conclusion}

The removal of radon-born lead contamination from organic ${ }^{65}$ liquid scintillator was studied in preparation of a low back-653 ground phase of the KamLAND experiment. Reduction factors were measured for various purification methods: water extraction with purified water and ion-enhanced water, isotope exchange, filtration, adsorption, and distillation.

Among these methods, distillation was identified as the most efficient method. It provides the reduction factors of $10^{3}$ $10^{4}$ required for KamLAND's solar neutrino detection phase. We obtained reduction factors for all components of the KamLAND LS: $n$-dodecane, pseudocumene and PPO. The studies performed on a scaled-up system provide the basic data for the design and operation of a purification plant for continuous circulation and purification of 1000 tons of KamLAND LS.

The second most effective procedure was silica gel extraction, which provided highly reproducible results. However, a steady decline observed in lead reduction factors after successive extractions cannot be explained by simple plate theory. One possible explanation is that a portion of the dissolved lead exists in non-polar organometallic compounds, which can be degraded by heat and/or chemically targeted on the lead-carbon bond. Our studies on silica gel extraction with preheating and processing with $\mathrm{FeCl}_{3}$ showed improved reduction factors consistent with the existence of such non-polar lead compounds in LS.

\section{Acknowledgments}

This work was supported in part by the U.S. Department of Energy (DOE) grant DE-FG02-01ER41166, the Japanese Ministry of Education, Culture, Sports, Science and Technology, the World Premier International Research Center Initiative (WPI Initiative), MEXT, Japan, DOE contract number DE-AC02-05CH11231, and other DOE grants from individual KamLAND institutions. The authors would like to thank Dr. Li Cang of Selecto Scientific Inc., and Xiong Xin Dai and Nick Jelly (Oxford University) of the SNO collaboration.

\section{References}

[1] J. Bahcall, Solar Neutrinos. I. Theoretical, Phys. Rev. Lett. 12 (1964) 300-302.

[2] J. Bahcall, R. Davis, Jr., Solar Neutrinos: A Scientific Puzzle, Science 1 (1976) 264-267.

[3] K. Eguchi, et al., First results from KamLAND: Evidence for reactor anti-neutrino disappearance, Phys. Rev. Lett. 90 (2003) 021802.

[4] T. Araki, et al., Measurement of neutrino oscillation with KamLAND: Evidence of spectral distortion, Phys. Rev. Lett. 94 (2005) 081801.

[5] S. Abe, et al., Precision Measurement of Neutrino Oscillation Parameters with KamLAND, Phys. Rev. Lett. 100 (2008) 221803.

[6] A. Gando, et al., Reactor On-Off Antineutrino Measurement with KamLAND, Phys. Rev. D 88 (2013) 033001.

[7] G. Keefer, First Observation of ${ }^{7}$ Be Solar Neutrinos with KamLAND, Ph.D. thesis, The University of Alabama, Tuscaloosa, Alabama, 2009.

[8] J. Bahcall, A. Serenelli, S. Basu, New solar opacities, abundances, helioseismology, and neutrino fluxes, Astrophys. J. 621 (2005) L85-L88.

[9] P. Collon, W. Kutschera, Z. Lu, Tracing Noble Gas Radionuclides in the Environment, Annu. Rev. Nucl. Part. S. 54 (2004) 39-67.

[10] Z. Djurcic, Measurement of reactor anti-neutrinos at KamLAND: Preparation and analysis, Ph.D. thesis, The University of Alabama, Tuscaloosa, Alabama, 2004. UMI-31-63548.

[11] C. Sutour, et al., Determination of the argon concentration in ambient dry air for the calculation of air density, Metrologia 44 (2007) 448-452. 
[12] J. Crank, The Mathematics of Diffusion, Oxford University Press, 2nd edition, 1975

[13] S. Takeuchi, Research and Development on Distillation-Purification for KamLAND Liquid Scintillator with respect to a ${ }^{7}$ Be Solar Neutrino Measurement, Master's thesis, Tohoku University, Sendai, Japan, 2005. Written in Japanese.

[14] L. Niedermeier, et al., Experimental scintillator purification tests with silica gel chromotography, Nucl. Instrum. Meth. A 568 (2006) 915.

[15] Y. Kishimoto, Liquid scintillator purification, AIP Conf.Proc. 785 (2005) 193-198.

[16] Y. Koseki, Study of liquid scintillator distillation for a low energy solar neutrino observation, Master's thesis, Tohoku University, Sendai, Japan, 2004. Written in Japanese.

[17] T. Ebihara, Study of liquid scintillator quality for a solar neutrino observation, Master's thesis, Tohoku University, Sendai, Japan, 2007. Written in Japanese.

[18] Y. Kibe, Purification of liquid scintillator by distillation for a solar neutrino observation, Master's thesis, Tohoku University, Sendai, Japan, 2007. Written in Japanese.

[19] M. Ogawa, Liquid Scintillator Purification for Solar Neutrino Observation with KamLAND: Studies on Lead Reduction by Distillation, Master's thesis, Tohoku University, Sendai, Japan, 2005. Written in Japanese.

[20] G. Keefer, Low background phase of KamLAND, Nucl. Phys. Proc. Suppl. 221 (2011) 358.

[21] G. Keefer, Laboratory Studies of Lead Removal from Liquid Scintillator in Preparation for KamLAND's Low Background Phase, AIP Conf.Proc. 1338 (2011) 175-178.

[22] H. Ogawa, Search for Electron Anti-Neutrinos from the Sun using the KamLAND Large Volume Liquid Scintillator Detector, Ph.D. thesis, Tohoku University, Sendai, Japan, 2003.

[23] A. Migdal, Ionization of atoms accompanying $\alpha$ and $\beta$-decay, J. Phys. (USSR) 4 (1941) 449.

[24] T. C. Andersen, et al., A radium assay technique using hydrous titanium oxide adsorbent for the Sudbury Neutrino Observatory, Nucl. Instrum. Meth. A 501 (2003) 386-398.

[25] J. Benziger, et al., A scintillator purification system for a large scale solar neutrino experiment, Nucl. Instrum. Meth. A 417 (1998) 278-296.

[26] L. S. Niedermeier, High Efficiency Purification of Liquid Scintillators for the Solar Neutrino Experiment Borexino, Ph.D. thesis, University of Technology, Munich, 2005.

[27] J. Benziger, et al., A scintillator purification system for the Borexino solar neutrino detector, Nucl. Instrum. Meth. A 587 (2008) 277-291.

[28] K. K. Unger, Porous Silica, its properties and use as support in column liquid chromatography, volume 16 of J. Chromatogr. Lib., Elsevier, New York, NY, 1979.

[29] D. Lide (Ed.), CRC Handbook of Chemistry and Physics, Sec. 3: Physical Constants of Organic Compounds, Chemical Rubber Publishing Company, Boca Raton, Florida, 89 edition, 2008-2009.

[30] D. S. Leonard, et al., Systematic study of trace radioactive impurities in candidate construction materials for EXO-200, Nucl. Instrum. Meth. A 591 (2008) 490-509.

[31] L. T. Zhuravlev, Concentration of Hydroxyl Groups on the Surface of Amorphous Silicas, Langmuir 3 (1987) 316-318.

[32] S. Tabuse, et al., Radiation protection effects by addition of aromatic compounds to n-dodecane, Radiat. Phys. Chem. 62 (2001) 179-187.

[33] B. K. Choudhuri, et al., Deleading of gasoline, Defense Sci. J. 11 (1961) 34-44.

[34] M. S. Kharasch, Process of deleading gasoline, US Patent 2453138 (1948).

[35] A. Furuta, K. Sato, K. Sato, JP Patent 97-170411 (1992).

[36] A. Deratani, B. Sebille, Metal ion extraction with a thiol hydrophilic resin, Analytical Chemistry 53 (1981) 1742-1746. 